\title{
Ho visto un sogno: Io assente ed es-terno in Omero
}

\author{
Emilio D’Agostino
}

Università degli Studi di Salerno

\section{Abstract}

L'articolo prende in esame la tipologia dei sogni che appare nei poemi omerici. Vi troviamo sogni ancora legati a quella che Freud definì "concezione primordiale», ossia una rappresentazione esterna al soggetto, una specie di messaggio inviato dagli Dei con fini esortativi o a volte per predire il futuro. Accanto a questi, appare già il sogno come attività della psiche del soggetto: è il caso del sogno di Penelope che anticipa la concezione moderna dell'attività onirica.

Parole chiave: genesi interna, genesi esterna, esortazione, predizione, desiderio.

\section{Abstract}

The article examines the type of dreams that appear in the Homeric poems. In them, we find dreams that are still inspired by what Freud defined as "primordial conception", or a representation outside the subject, as a type of message sent by the Gods as an exhortation or at sometimes to predict the future. As well as these, the dream also appears as an activity by the subject's psyche: this is true of Penelope's dream, which anticipates the modern conception of dream activity.

Key words: inner genesis, outer genesis, exhortation, prediction, desire.

[...] Architecte de me féeries/je faisais, à ma volontè,/sous un tunnel de piérreries/passer un océan dompté;/et tout, même la couleur noire,/semblait fourbi, clair,irisé;/le liquide enchâssait sa gloire/dans le rayon cristallisé [...] 


\section{L'interesse di Freud per la filosofia antica: l'interpretazione dei sogni}

Le metafore archeologiche ${ }^{1}$ spesso usate, così come il riferimento al personaggio di Edipo della tragedia di Sofocle, nella costruzione della prima fase ${ }^{2}$ del pensiero psicoanalitico, testimoniano dell'interesse di Sigmund Freud verso il mondo antico in generale e, più in particolare, verso la filosofia greca. Sotto l'influenza della Jung-Wien e del Circolo di Basilea, ${ }^{3}$ Freud si avvicina al mondo greco e ne trae suggestioni significative.

E' in particolare in relazione al tema dell'analisi dei sogni e della loro interpretazione che lo studioso di origine morava si apre alla tradizione antica. Oudai Celso (2006) mette in luce come:

proprio l'analisi di fenomeni onirici — nella quale l'autore riponeva i più fervidi auspici di riconoscimento e notorietà, a dispetto delle impietose stroncature iniziali- costituisce l'occasione di un sistematico e cospicuo sforzo di documentazione volto a reperire proprio nell'antichità, filosofica e non solo, gli spunti per un'indagine onirologica rigorosamente impostata, ma la tempo stesso capace di oltrepassare quel riduzionismo scientista incline a relegare il sogno nel dominio dei fenomeni puramente fisiologici, negandogli ogni valenza psichica. ${ }^{4}$

E in tale direzione va l'apertura del saggio del 1899 Traumdeutung (L'interpretazione dei sogni) nella quale Freud distingue tra la concezione "primordiale» dei greci e quella che successivamente verrà elaborata a partire dal V sec. a.C. Questo secondo riferimento è, naturalmente, in primo luogo a Platone e ad Aristotele.

La concezione che Freud chiama "primordiale» è contraddistinta da una definizione del sogno come puro messaggio inviato durante il sonno a un individuo da parte della divinità. Esso non è, infatti, prodotto dell'individuo stesso,

1. Freud non esita a paragonare il lavoro dell'analista a quello dell'archeologo, evidenziando la relazione tra il suo metodo analitico e il tradizionale metodo dell'anamnesi, cioè la ricostruzione scientifica del passato, così come Schliemann aveva fatto nel caso di Itaca, di Micene e dell'antica Troia. Cfr. Sigmund Freud, Zur Ätiologie der Hysterie, Wiener klinisce Rundschau, vol. 10, 1896. Trad. it.: Etiologia dell'Isteria, vol. 2, Opere Complete, Milano: Bollati Boringhieri, 2007, p. 334.

2. Il ruolo del «complesso di Edipo» (denominato anche "complesso nucleare»), definizionale nel periodo tra i tre ed i cinque anni del bambino e definito dal desiderio della morte del genitore dello stesso sesso e dal desiderio sessuale nei confronti del genitore dell'altro sesso, è fondamentale nell'edificio freudiano e, in questo, il suo superamento è determinante nella cosiddetta «scelta oggettuale» in età adulta. Esso, in effetti, costituisce, nella definizione della sessualità infantile, la base per il riconoscimento della costituzionale bisessualità dell'individuo. Nello sviluppo successivo del pensiero analitico, come nel caso di Melanie Klein, nella fase "pre-edipica» è riconosciuto, invece, il ruolo determinante della madre.

3. Il riferimento è al circolo politico, giornalistico e letterario che riuniva personalità come A. Schnitzler e H. Von Hoffmanstal ed al gruppo di intellettuali e docenti dell'Università di Basilea come F. Nietzsche e J. Burkcardt.

4. Cfr. Yamina Oudai CELSO, Freud e la filosofia antica, Milano: Boringhieri, 2006, p. 58. 
ma costituisce una pura «visione» generata volontariamente dall'esterno. Veritiera oppure ingannevole essa assume, in genere, un carattere "esortativo», spingendo il soggetto passivo dell'attività onirica ad un'azione: quella voluta o incoraggiata dalla divinità. Allo stesso tempo, il contenuto del sogno, spesso oscuro, come il linguaggio dell'oracolo, è incomprensibile al soggetto sognatore passivo, quindi, richiede l'intervento di un oneirokrites («interprete dei sogni»). ${ }^{5}$

Se il sogno è equiparato, in termini freudiani, al sintomo nevrotico, al lapsus e ai cosiddetti "atti falliti», in quanto espressione del conflitto tra desiderio e censura nella psiche di un individuo, ${ }^{6}$ il primo antecedente nella storia del pensiero greco è rappresentato dalla Repubblica di Platone, in particolare il Libro IX. Ed è in questo riconoscimento che probabilmente giace il debito freudiano verso la filosofia greca e, allo stesso tempo, il superamento stesso nei confronti di questa. All'inizio del Libro IX della Repubblica, Platone parla esplicitamente di desideri irrefrenabili "particolarmente violenti e numerosi» che si scatenano nel sonno in assenza di inibizioni:

[Socrate] «E questi desideri quali sarebbero?», domandò. «Quelli che si risveglianodurante il sonno - risposi- quando tutto il resto dell'anima dorme - con ciò intendo riferirmi alla sua parte razionale, moderata e predominantee invece salta fuori quella animalesca, selvatica, che si riempe di cibo e di bevande; e questa, facendosi largo nel sonno cerca di venire a galla e soddisfare le sue aspirazioni. Del resto, tu non ignori che in tali condizioni essa osa fare di tutto come se fosse libera da ogni remora imposta dal pudore e dalla saggezza. Così, ad esempio, non ha alcuna esitazione a rappresentarsi un'unione con la madre, o con un altro uomo, qualsiasi sia, o con dei o con animali, oppure a macchiarsi del sangue di chiunque, o a cibarsi di qualsiasi cosa. Insomma, non lascia indietro nulla per folle e indecente che sia»?

Tale passo consente a Jaeger di riconoscere in Platone «il padre della psicoanalisi»:

Nel sogno, quando, l'anima si libera dalle catene delle inibizioni impostele dalla ragione, e nell'uomo si risveglia l'elemento selvaggio e ferino, si rivela questa parte della sua natura, il più delle volte sconosciuta all'uomo stesso. Platone è il padre della psicoanalisi. E' il primo che abbia svelato come parte dell'io subcosciente la mostruosità del complesso di Edipo, del desiderio del-

5. Per tale aspetto, cfr. ARTEMIDORo Di DALdi, 'Oneirokritiká, trad. it.: Giulio GuidorizzI (a cura di), Il libro dei sogni, Milano: Biblioteca Universale Rizzoli, 2006.

6. Il sogno per Freud è allo stesso tempo un prodotto psichico, una forma di pensiero resa possibile dallo stato di sonno, è la forma nella quale pensieri scartati dal preconscio o dalla stessa coscienza in condizione di veglia riemergono nelle condizioni favorevoli date dal sonno, ha un'organizzazione linguistica fondata sullo spostamento e la condensazione (come la metafora), è il cammino verso la conoscenza dell'incoscio — non è l'inconscio- ed è una modalità di appagamento di un desiderio rimosso. Tale carattere possiede il tratto della generalità, meno nei casi dei sogni di punizione, di controdesiderio e nei sogni di angoscia.

7. Cfr. Platone, Repubblica, L. IX 571 A, in Giovanni Reale, Opere Complete, Milano: Bompiani, 2006. 
l'unione sessuale con la madre, valendosi, per metterla in luce, dell'esperienza del sogno, e indicando anche complessi similari di desideri repressi che arrivano fino all'unione sessuale con divinità, alla sodomia, alla libidine dell'assassinio. $^{8}$

In realtà, va notato come alcuni ostacoli insormontabili si presentino al momento della definizione di un'associazione stretta, quasi vincolante, Platone-Freud. In primo luogo: anche se in maniera del tutto evidente c'è similitudine tra quanto sostenuto da Platone e da Freud in relazione alla dinamica tra desiderio represso e sogno, la cornice nella quale si muove il primo è di tipo politico-morale con una chiara valenza pedagogica, mentre il secondo si dirige verso un'interpretazione di tipo strettamente psichico. Infatti, il discorso platonico si svolge nella Repubblica a proposito della figura del «tiranno» e del "democratico».? In secondo luogo: poiché la dimensione morale e quella pedagogica sono proprie dello scritto platonico, l'obiettivo posto dall'ateniese è il Bene assoluto, ${ }^{10}$ raggiungibile attraverso il prevalere della ragione e della moderazione, invece la posizione freudiana è di tipo esclusivamente naturalistico, poiché riconduce e riduce ogni interpretazione alla condizione della "natura» umana. Essa è scientifica e non morale. D'altro lato, «psiche» e pychè non sono nozioni per nulla assimilabili. Se la pychè omerica altro non è che «respiro» che fugge via al momento della morte, la rappresentazione platonica, mai esplicita e mai diretta, ${ }^{11}$ è nel Fedro, ad esempio, quella dell'auriga e i due cavalli, uno bianco e docile e uno nero e ribelle, il cui controllo è necessario. ${ }^{12}$ Inoltre, la costruzione teorico-filosofica freudiana si muove per definizione in un'ottica di negazione rispetto agli atteggiamenti di tipo razionalistico, come ad esempio, quello di tipo illuministico che ponevano come necessario il superamento delle inclinazioni individuali e private, allo scopo, ancora una volta, di

8. Cfr. Werner Jaeger, Paideia. Die Formung des griechischen Menschen, Berlin-Leipzig: W. De Gruyter \& Co., 1944. Trad. it.: Giovanni Reale (a cura di), Paideia. La formazione dell'uomo greco, Milano: Bompiani, 2003, p. 1287.

9. In relazione al Platone «politico», rimando al saggio del 1937 di Giorgio Colli, in cui è esaminata l'evoluzione del pensatore ateniese in rapporto alla politica, così come la sua posizione "anti-democratica» più volte richiamate in letteratura, come nel più recente saggio di Dunn (2005). Cfr. Giorgio Colli, Platone politico, in Nuova rivista storica, ripubblicato in Giorgio Colli, Platone politico, Milano: Adelphi, 2007; e John DunN, Setting the People Free. The Story of Democracy. Trad. it.: Il mito degli uguali. La lunga storia della democrazia, Milano: Univ. Bocconi.

10. A tale riguardo, cfr. Giovanni ReAle, Corpo, anima e salute. Il concetto di uomo da Omero a Platone, Milano: R. Cortina Ed., 1999, cap. 17.

11. Cfr. a tale riguardo Giovanni REALE, op. cit. In realtà, io credo che la riluttanza di Platone a definire in modo esplicito il concetto di «anima» e, quindi, il suo uso di metafore come quella citata, sia nel Fedro, come nella Repubblica, si debba probabilmente alla stessa remora profonda espressa nella cosiddetta «Settima lettera» sul linguaggio verbale e sulla scrittura in particolare. A tale riguardo e in merito alla "mania», Cfr. Giorgio Colli, La nascita della filosofia, Milano: Adelphi, 1975; in merito alla "mania».

12. Sui miti platonici rimando a Franco Ferrari (a cura di), I miti di Platone, Milano: BUR, 2006. 
raggiungere verità razionali universali. ${ }^{13}$ In terzo luogo: la forza complessiva del desiderio freudiano, la libido ("l'energia corrispondente all'aspetto psichico della pulsione sessuale»), più che ad una natura irrefrenabile, ma di tipo puramente irrazionale, si approssima maggiormente alla definizione platonica di eros del Simposio. ${ }^{14}$

Nella sua rivisitazione delle concezioni del sogno proprie dell'età classica, nel Traumdeutung, Freud cita direttamente le posizioni di Aristotele espresse nel De divinatione per somnium e nel De somniis. Lo stagirita, infatti, partendo da una definizione del sogno come «attività psichica propria dell'uomo addormentato", nella sua apparente semplicità, riconduce la produzione onirica al suo carattere attivo, individuale e interno al soggetto:

Nei due scritti di Aristotele sul sogno [...] esso è già diventato oggetto della psicologia: non è inviato dalla divinità, e la sua natura non è divina, ma demoniaca, dato che la natura è demoniaca e non divina. In altre parole, esso non proviene da una rivelazione soprannaturale, ma dalle leggi dello spirito umano, che però è affine alla divinità. ${ }^{15}$

Su tale base, nell'analisi di seguito proposta della rappresentazione omerica dell'attività onirica, sono, a mio avviso, da evidenziare due fondamentali questioni e ciò̀: a) la compresenza di una duplice rappresentazione del sogno, sia in termini di concezione "primordiale» sia in termini strictu sensu di attività psichica, rappresentazione volta, in entrambi i casi, allo sviluppo narrativo dei due poemi; b) la considerazione aristotelica della natura demoniaca del sogno analoga a quella della stessa natura.

A tale scopo prenderò in esame caratteri dei principali sogni espliciti presenti nella narrazione omerica.

\section{Le narrazioni omeriche e il sogno nei poemi}

E' per me certamente impossibile riportare qui la bibliografia più significativa sulla cosiddetta "questione omerica» che, in quanto tale, comunque non sarebbe che di importanza marginale, né tantomeno la bibliografia sulle due tradizioni narrative che hanno nome Iliade e Odissea. Mi preme, però, ricordare il loro carattere composito, stratificato e diversificato, ma, soprattutto, in quanto tale, rappresentativo delle idee che il lungo periodo storico-culturale greco che definiamo "omerico» ebbe di sè e che in parte presenta tratti che, nello stesso linguaggio comune di oggi, si manifestano, più che come residui o reperti archeologici, come "grumi» discorsivi consolidati tuttora presenti e vivi.

13. Cfr. Eli Zaretsky, Secrets of the Soul. A social and Cultural History of Psychoanalysis, New York: Alfred A. Knopf. Trad. it.: I misteri dell'anima. Una storia sociale e culturale della psicoanalisi, Milano: Feltrinelli, 2004, cap. 1.

14. Cfr. Oudai Celso, op. cit. e Giovanni Reale, op. cit.

15. Cfr. Sigmund FreUd, op. cit., p. 12. 
Poiché entrambe le tradizioni discorsive, Iliade e Odissea, forniscono una piena rappresentazione fondamentalmente omogena e completa circa la natura esterna di «sentimenti» e «emozioni», come della loro collocazione nel corpo dell'uomo e della fisicità dello stesso linguaggio, ${ }^{16}$ quattro questioni mi paiono qui determinanti. La prima: Menis e Metis. Se il poema Iliade si identifica fondamentalmente con la rappresentazione di un eccesso, l'ira, la menis ${ }^{17}$ in tutte le sue fasi e sfumature, il poema Odissea, al contrario, si identifica fondamentalmente con l'astuzia, la metis, o forse meglio "l'astuzia della ragione» e nell'uso determinato dell'inganno e della menzogna, al «rapporto di due rapporti» nella "coscienza menzognera» di Jankélévitch. ${ }^{18}$ I due personaggi dominanti, Achille e Ulisse, si affidano il primo alla dimensione passionale anche nelle sue stesse proprietà fisiche e il secondo, invece, alla dimensione del ragionamento avvolgente e dell'uso controllato e finalizzato del linguaggio. La secon$d a$ : Io maschile e Io femminile. Se con Achille si realizza, forse, la prima manifestazione di un Io maschile non più eterodiretto, con Penelope - specchio femminile di Ulisse nella metis — si manifesta un'analoga manifestazione di un altro Io non più eterodiretto: quello di una donna. La terza: sogni diversi. Il carattere composito e stratiticato dei poemi omerici è tale che, in essi, possano coesistere concezioni e rappresentazioni diverse ed autonome del sogno. La quarta: il Tempo e la Finzione. Se l'Iliade è il nostro primo grande «discorso»

16. Cfr. a tale proposito Anna Marina Storoni PiAzZA, Ascoltando Omero. La concezione di linguaggio dall'epica ai tragici, Roma: Carocci, 1999. Inoltre, mi pare importante notare che la dimensione fisica del linguaggio, e di quanto successivamente sarà per molta parte definito logos, sia propria anche della stessa tradizione biblica. Si veda, dunque, Adriana CAVARERO, A più voci. Filosofia dell'espressione vocale, Milano: Feltrinelli, 2003. In particolare, per i termini ruah («fiato», «respiro») e qol («voce»), ad esempio nella Genesi e nel Salmo 33. Non solo la creazione avvenne con il respiro divino, ma la Sua «voce» per divenire linguaggio articolato dovette attendere i profeti. Inoltre, non è un caso che "profeta", oltre a significare "colui che viene prima» (ad esempio il Battista), significhi anche "colui che parla in nome di». Tale aspetto, l'esser in primo luogo «fisicità» e «natura», sarà solo molto più tardi pienamente ripreso da Giovambattista Vico. Tale tratto che è certamente definizionale per il linguaggio umano ha una serie di conseguenze su piani diversi ma, ritengo, attualmente soprattutto sul quello dell'etica del discorso. Rimando per tale ultima questione a Emilio D’Agostino, Fondamenti linguistici dell'etica, in Riscontri, XXIX, 1, gennaio-marzo, p. 19-43; e a Emilio D’Agostino, Comunicazione e informazione. Sans toit ni loi?, Dipartimento Scienze della Comunicazione, Unisal, 2007.

17. A tale riguardo rimando a Sergio MORAVIA, Emozione e passione, in Silvia VegETTI FINZI, Storia delle passioni, Bari: Laterza, 1995, p. 3-38; e a Emilio D’AGOSTINO, "Grammatiche lessicalmente esaustive delle passioni. Il caso dell'Io collerico. Le forme nominali», Quaderns d'Italià, n. 9, 2004.

18. Sull'Ulisse polymechanos, rimando a Andrea TAGLIAPIETRA, La filosofia della bugia. Figure della menzogna nel pensiero occidentale, Milano: Mondadori, 2001; e a Vladimir JANKÉLÉVITCH, Du mensonge, Paris: Flammarion, 1998; edizione italiana: La menzogna e il malinteso, Milano: R. Cortina Ed., 2000. Per il rapporto tra «fini» e «mezzi» si veda il breve e divertente David Herzog, Cunning, Princeton Univ. Press, 2006. Trad. it.: Furbate, Milano: Rizzoli, 2006. Per la questione della descrizione del lessico e della grammatica della e sulla menzogna rinvio a Emilio D’AGOSTINO, «Per una grammatica lessicalmente esaustiva sull'inganno e la menzogna», Quaderns d'Italià, n. 8, 2004. 
sulle passioni, l'Odissea lo è della memoria e della nostalgia, come del (continuo) ritorno. L'Odissea è, allora, il poema del Tempo, come nota Harold Bloom:

C'è un elemento, nell'Odissea, che è già quasi virgiliano: il senso delle cose nel loro trascorrere. ${ }^{19}$

e, contemporaneamente, con Mario Lavagetto:

[...] se il mondo dell'Odissea è [...] un mondo di isole, chi viene da lontano, dal mare, da altre terre e altre isole, chi è "nessuno", può essere "chiunque»: alle sue spalle non ci sono testimoni e la sua libertà di narrazione e di falsificazione è completa. ${ }^{20}$

Outis: Nessuno. Potenzialmente, quindi, tutti e chiunque. Frutto di quel particolare discorso di quel preciso momento, luogo dell'invenzione. Come i racconti del polutropos Odysseus, ricco di espedienti. Della «menzogna» di ogni narrazione: il luogo della letteratura. Il luogo del racconto, ancorchè doloroso:

[Odisseo, Libro XII, vv. 450-453] Ma perché lo racconto? Già ieri questo ho narrato, qui in sala, a te e alla tua forte sposa: m'è odioso narrare di nuovo cose già dette.

Infine, la condizione del proto-bugiardo e di Wilde. Dell'inutile e di Bataille. Allo stesso tempo, però: se chiunque, come Odisseo, è straniero, chiunque è anche colui dell'esilio, il «barbaro», il «balbettante», colui che non parla bene la lingua delle terre cui giunge. ${ }^{21}$

\subsection{Iliade e Odissea. Sogni maschili, sogni femminili ${ }^{22}$}

Nei poemi omerici, diverse sono le rappresentazioni e le manifestazioni del sogno. Alcune di esse sono esplicite e chiare, altre sono semplicemente indirette. Nel primo caso, si tratta del racconto diretto di un sogno di un personaggio, nell'altro, invece, si ha a che vedere con atmosfere che possono ricordare quella propriamente onirica, ${ }^{23}$ oppure situazioni nelle quali è richiesto l'in-

19. Cfr. Harold Bloom, Genius. A Moisaic of the One Hundred Exemplary Creative Minds, 2002; trad. it.: Il Genio, Milano: Rizzoli, 2004, p. 586.

20. Cfr. Mario Lavagetto, La cicatrice di Montaigne. Sulla bugia in letteratura, Milano: Bompiani, 2002, p. 9.

21. Sulla questione de «l'esilio» e della relativa condizione umana, rimando a Josip BRODSKIJ, The condition We Call Exile, 1988; trad. it.: Dall'esilio, Milano: Adelphi, 1998; e a Elias CANETTI, Die gerettete Zunge. Geschichte einer Jugend, München, C. Hanser Verlag, trad. it.: La lingua tagliata. Storia di una giovinezza, Milano: Adelphi, 1991.

22. Ringrazio la dott.ssa Paola Sonderegger della Biblioteca provinciale di Salerno, per l'aiuto datomi nella revisione e nel commento delle traduzioni italiane dei due poemi omerici.

23. Tale compresenza, congiuntamente ad altri aspetti, spinge lo psicoterapeuta adleriano F. Maiullari a sostenere che l'Iliade sia un poema scritto "come se fosse un sogno», scritto 
tervento di un oneiropolos o oneirokrites, cioè di un interprete dei sogni. Presagi, profezie, responsi: cari ad Apollo/Dioniso, ${ }^{24}$ ingannevoli o veritieri, ma sempre discorsi dell'ambiguità. ${ }^{25}$

In questo contributo, io mi occuperò dei principali racconti espliciti di sogni, riservandomi per una prossima occasione l'analisi di possibili riferimenti testuali non di tale tipo. ${ }^{26}$

\subsection{Iliade: il sogno di Agammenone (L. II vv. 5-34). \\ Desiderio di rivincita e desiderio di vittoria}

Nell'iliade, il primo sogno è quello del basileus basileuteroi: Agamennone. Com'è noto, si tratta del sogno ingannatore, cattivo o funesto (oulos oneiros) inviato da Zeus ad Agammenone. Il messaggero non è la riproduzione in immagine perfetta, ma è, per definizione, solo simile, nelle forme, al figlio di Neleo. ${ }^{27}$ Oulos oneiros: qui Omero anticipa la classificazione dei sogni che sarà esplicitata solo molto più tardi nell'Odissea da Penelope nell'incontro con Ulisse che non le ha ancora svelato la propria identità. La teoria delle "due porte», su cui tornerò più avanti, nel commento allo stesso sogno di Penelope. Tale classificazione è preceduta, però, nel Libro I dell' Iliade dalla prima definizione esplicita della natura dei sogni. Essa è divina:

anche il sogno è da Zeus [Iliade, Libro I, v, 63]

ed è fornita da Achille, nell'assemblea dei capi achei, che si interrogano sulle ragioni dell'ira di Apollo. Nel Libro II, il racconto dell'invio del sogno ad Agammenone apre la narrazione. Lo stesso Zeus, molto umanamente, non può addormentarsi, finchè non prende la decisione che gli appare la più «bella». Zeus, attraverso il sogno, spinge Agamennone ad un nuovo attaco alle mura di Troia che si rivelerà, com 'è noto, quasi fatale per l'esercito acheo. Vanno

"come se fosse un racconto onirico» o «un fantasma oniroide» o «un'affabulazione in stato sognante». Cfr. Franco MaIUlLARI, Omero anti-Omero. Le incredibili storie di un trikster giullare alla corte micenea, Roma: Ed. Ateneo, 2004.

24. Per il rapporto Apollo/Dioniso e la critica a Nietzsche che vede antitesi tra le due divinità, si veda ancora Giorgio Colli, op. cit.

25. La stessa profezia di Tiresia, "l'oracol mio che non tinganna», ad Ulisse nell'Ade (Odissea, L. XI, vv. 158-183), si muove sul piano dell'ambiguità (Morte dal mare ti verrà, molto dolce, a ucciderti vinto da una serena vecchiezza), anche se questo è il passo che consente ad Harold Bloom di riproporre la visione di un «unico» Omero nata con l'ellenista Longino. E', inoltre, la stessa profezia necessaria alla rappresentazione dantesca della morte di Ulisse oltre le colonne d'Ercole condannato per il peccato di hybris (Inferno, Canto XXXIV, vv. 106-120). Cfr. Bloom op. cit.

26. Mi riferisco qui, in particolare, al «sogno» di Priamo prima di recarsi al campo di Achille per ottenere la restituzione del corpo di Ettore e a quello di Achille nel quale appare Patroclo che gli chiede la sepoltura necessaria per entrare nell'Ade.

27. Nestore avrà un ruolo fondamentale nello sviluppo successivo, in quanto nel "gioco di rinforzo» con Agamennone, darà credibilità al racconto fatto dall'Atride, prima dell'intervento dell'interprete dei sogni. 
notate subito due questioni: il ruolo di Agamennone basileus, da un lato, e, dall'altro, la funzione narrativa del suo sogno. Per la prima, è sufficiente richiamare E. Benveniste sugli attributi e i doveri della regalità indo-europea: l'Atride, per quanto concerne il geras («bottino») ${ }^{28}$ è venuto meno ai suoi doveri, sottraendo ingiustamente Briseide ad Achille, in cambio della restituzione di Criseide al padre. E' venuta meno la funzione di equo «ripartitore» dei frutti della guerra tra il suo popolo. Non è stato più, direbbe Canetti, il «fecondatore». ${ }^{29}$ Inoltre, ha commesso il più grave peccato che un un uomo possa commettere e cioè si è abbandonato alla hybris, offendendo Apollo attraverso il suo "portavoce» Crise che, come tale, si presentava al campo acheo, portandone le insegne (lo skeptron). Crise aveva parlato «in nome di» e come tale andava rispettato e non minacciato e scacciato. Quindi, Agamennone è doppiamente colpevole e, dunque, da punire. La punizione lo colpirà due volte: sotto le mura di Ilio il suo desiderio di rivalsa su Achille non troverà soddisfazione, anzi porterà quasi alla distruzione del suo esercito e, dopo, al suo ritorno in patria, nelle tragedie di Euripide e di Sofocle, ucciso da Egisto o da Clitennestra, come da una freccia venuta da lontano e della quale non è possibile sapere quando e da chi è stata scoccata. Per la seconda: Maiullari annota la necessità narrativa di questo sogno nell'impianto circolare del poema. Dunque: l'Iliade ha inizio con la menis oppure con questo oneiros "cattivo»? ${ }^{30}$ Più che verosimile la seconda risposta.

E', dunque, in occasione della rappresentazione del sogno di Agamennone che appaiono due espressioni fondamentali per la comprensione di questa concezione. Si tratta dello uper kephales e dell'uso della forma verbale idein («vedere») associata a onar: il sogno — cioè il messaggero della divinità- si colloca sulla testa del dormiente e questi «lo vede». Se entrambe le espressioni contribuiscono a definire la dimensione esterna dell'attività onirica e la conseguente passività del sognatore, attraverso la fisicità manifesta del sogno, la seconda, in particolare, richiama particolarmente l'attenzione. Certamente rientra la fisicità necessaria alla vista — come in una rappresentazione teatrale - ma, l'uso di idein aggiunge un di più necessario alla comprensione dell'uomo greco, non solo omerico: il tema della vista e del vedere. Da Snell ${ }^{31}$ in poi, tale aspetto è stato preso spesso in considerazione. Registrata la pluralità dei verbi lega-

28. Sulla questione del geras greco e del potlac polinesiano, cfr. Emile BENVENISTE, Le vocabulaire des institutions indoeuropéennes, vol. I, Economie, parenté, societé, cap. 2, Paris: Ed. Minuit, trad. it.: Il vocabolario delle istituzioni indoeuropee, vol. I, Economia, parentela, società, Torino: Einaudi, 1981; e Marcel MaUss, Essai sur le don. Formes et raison de l'échange dans les societés archaïques, in L'année sociologiques, I, p. 30-186, 1923-24, trad. it.: Il saggio sul dono. Forma e motivo dello scambio nelle società arcaiche, Torino: Einaudi, 2002.

29. Sugli attributi regali come manifestazione del potere, cfr. Elias CANETTI, op. cit., in particolare, le parti relative a Gli organi del potere e Sovranità e paranoia.

30. Franco MaIUllari, op. cit.

31. Rimando a Bruno SNELL, Des Entdeckung des Geistes. Studien zur Entstehung des europäischen Denkens bei den Griechen, Hamburg: Claassen Verlag, trad. it.: La cultura greca e le origini del pensiero europeo, Torino: Einaudi, 1963, in particolare il cap. I. 
ti a tale dominio, nonostante la riduzione degli usi in epoca post-omerica, il senso della vista rappresenta in Grecia antica «il senso per eccellenza», quello più profondo, più veritiero e anche quello più pericoloso (il farsi vedere). Su tale strada, Franzoni (2006), può affermare che «l'uomo greco è nato per vedere» e che ci si trova dinanzi ad una "tirannia dello sguardo». La visione degli dei (e dei loro messaggeri) è l'emozione più importante, ${ }^{32}$ essa è conoscenza e sorgente di pericoli. ${ }^{33}$ D'altronde, la cecità è da sempre temuta. La visione, da ultimo, è fonte e strada di Eros, anche più tardi come con messer notaro Jacopo da Lentini:

Amor è un desio che vien da core/per abbondanza di gran piacimento;/e li occhi in prima generan l'amore/e lo core li dà nutricamento./Ben è alcuna fiata om amatore/senza vedere so 'namoramento,/ma quell'amor che stringe con furore/da la vista de li occhi ha nascimento

Gongora di vista e cecità, quindi del ruolo dell'occhio, nella «tirannia amorosa» dirà nel testo del 1580 :

Ciego que apuntas y atinas, caduco, dios y rapaz/vendado que me has vendido y niño mayor de edad/con el alma de tu madre que murió siendo inmortal/de envidia de mi señora que no me persigas más/que no me persigas más /Déjame en paz amor tirano, déjame en paz/Amadores desdichados que seguís milicia tal/decidme que buena guía podéis de un ciego sacar/de un pájaro que firmeza que esperanza de un rapaz/que galardón de un desnudo de un tirano que piedad. ${ }^{34}$

Il greco omerico, nell'Iliade, infatti, parla non di «avere un sogno» o di "fare un sogno", bensì di "vedere un sogno": onar idein. ${ }^{35}$ In tale formulazione, il predicato onar associato all'uso di idein, richiama il carattere esterno all'inconscio e la passività del soggetto sognatore. La struttura linguistica sembra riconducibili ad una frase con subordinazione completiva del tipo: Soggetto umano vedere che Frase subordinata.

32. Cfr. David Le Breton, La saveur du monde. Une anthropologie des sens, Paris: Ed. Métallié, trad. it.: Il sapore del mondo. Un'antropologia dei sensi, Milano: R. Cortina. Ed., 2007.

33. Cfr. Claudio Franzoni, Tirannia dello sguardo. Corpo, gesto, espressione dell'arte greca, cap. II e III, Torino: Einaudi, 2006.

34. Cfr. Luis de GÓNGORA, Obras Completas, Madrid.

35. Ringrazio A. M. Langella per avermi fatto notare come anche in italiano contemporaneo quest'uso di vedere si manifesti con continuità in espressioni del tipo ho visto giusto, ha una visione corretta di ciò e nei segnali discorsivi a clichè di presa di turno nella conversazione come veda. La stessa tradizione del vedere un sogno, ancora, è propria della cabala napoletana e dell'interpretazione dei sogni di numeri. In Non ti pago, Edoardo, pur nel gioco dell'ironia, a questa si riferisce. 


\subsection{Odissea. Sogni al femminile}

Come l'Iliade è identificabile (in parte) con il semidio Achille, l'Odissea lo è con il mortale Ulisse. All'illusione dell'eroicità:

[Achille, Odissea, Libro 11, vv. 487-492, l'evocazione dei morti] Non lodarmi la morte, splendido Odisseo, vorrei essere bifolco, servire un padrone, un diseredato, che non avesse ricchezza, piuttosto che dominare su tutte l'ombre consunte.

è contrapposta la certezza del cambiamenento ottenuto grazie alla continua separazione, al distacco, alla discontinuità. Per dolorosi e strazianti che essi possano essere. Alla menis funesta, segue la metis ingegnosa e, in contrasto, si legge:

Menin aeide, theà, Peleiadeo Akileos (Iliade)

Andra moi ennete, Mousa, polytropon (Odissea)

Poco prima, però, s'è detto che l'Odissea è la narrazione del tempo, dell'invenzione, della nostalgia e della memoria, ma, forse, costituisce anche il primo grande "discorso" sulla sessualità femminile. Anzi, per certi aspetti, è proprio la sessualità femminile che accompagna tutto lo sviluppo del poema. Escluse le divinità femminili come le maghe e ninfe - l'invitante Circe e Calipso riccioli belli- restano in primo piano per l'argomentazione che qui m'interessa soprattutto Nausicaa e Penelope ${ }^{36}$ In particolare, sarà la seconda a rivelare tratti di originalità assoluta sia sul piano della rappresentazione oniri$\mathrm{ca}$, sia su quello più generale dell'affermazione di un Io femminile.

\subsection{Odissea. Sogni d'amore. Il sogno di Nausicaa (L. VI vv. 10-47)}

La glaucopide Atena, come un soffio di vento, mosse verso la stanza ornata in cui una fanciulla dormiva, per favorire il ritorno di Ulisse: sembrando la figlia di Dímante, stette sulla testa (uper kephales) della giovane. Nella preparazione del sogno, nulla appare diverso dai sogni maschili dominanti nell' Iliade. L'invito a risvegliarsi altro non è che la preparazione all'incontro con Ulisse, ormai trascinato sulla sua zattera fino alla spiaggia dei Feaci. Nausicaa peplo bello, risvegliatasi, accoglie l'esortazione della dea e si dirige con le sue ancelle verso il luogo dell'incontro inatteso. L'inganno teso dalla dea, pur non trattandosi di un «sogno funesto", come nell'episodio di Agammenone, è servito soltanto a preparare l'incontro con Ulisse e, di qui, la sua ripartenza verso Itaca.

36. La benevolenza delle donne verso Odisseo, siano esse divinità o mortali, spinge verso l'associazione della sua figura a quella del puer aeternus nella polarità archetipale di tipo junghiano Senex-Puer: necessità continua di cambiamento, irrefrenabile spinta al «viaggio», alla curiosità, all'inventiva e alla prometeica capacità d'inganno. Per tali aspetti, cfr. James HiLlman, Senex and Puer. An Aspect of the Historical and Psychological Present, 1967, trad. it.: Puer aeternus, Milano: Adelphi, 1999. 
In realtà, l'esortazione della dea avviene mediante l'immagine di una giovane amica e in nulla appare diversa da quelle analoghe mandate ai personaggi maschili dominanti nell' Iliade. Il possibile desiderio amoroso risvegliato di Nausicaa servirà allo scopo. In sintesi, il sogno della giovane Nausicaa ha la stessa strutturazione del sogno di Agamennone: si tratta di due varianti dello stesso tema e della stessa concezione.

\subsection{Odissea. Sogni di donna. Il sogno di Penelope e la teoria del sogno (L. XIX vv. 505-569)}

In modo non prevedibile si sviluppa, invece, l'episodio del sogno di Penelope. L'anomalia, infatti, è data dalla genesi stessa del sogno e dai suoi contenuti. In apparenza, esso costituisce soltanto un sogno particolarmente oscuro che, per essere interpretato ha bisogno di un interprete, un oneirokrites, e di cui la natura è ignota allo stesso soggetto sognatore, Penelope. La donna, infatti, non fa che essere ancora portatrice della concezione tradizionale dell'attività onirica. E non potrebbe essere altrimenti. Nel colloquio con Odisseo, che ancora non le ha svelato la propria identità, Penelope riprende la teoria delle «due porte»:

Ospite, i sogni sono vani, inspiegabili: non tutti si avverano, purtroppo, per gli uomini. Due sono le porte dei sogni inconsistenti: una ha battenti di corno, l'altra d'avorio: quelli che vengono fuori dal candido avorio, avvolgon d'inganni la mente, parole vane portando; quelli che invece escon fuori dal lucido corno, verità li incorona, se un mortale li vede. (L. XIX vv. 505-569)

Si può, dunque, notare: la ripresa dell'espressione onar idein, il carattere ingannevole o veritiero del sogno, la sua opacità e, infine, la sua inconsistenza materiale. Ancora una volta, il sogno altro non è che un messaggio giunto dall'esterno come possibile predizione e il soggetto sognatore è un soggetto passivo. In realtà, ciò è soltanto quanto ritiene la stessa Penelope, poiché alcune caratteristiche del suo sogno presenti nella sua stessa narrazione ad Ulisse spingono in una direzione diversa. ${ }^{37}$ In sintesi: il sogno di Penelope è rappresentato dal sopraggiungere improvviso di una grande aquila dal becco adunco che strazia le oche di Penelope che le stanno beccando il grano nel cortile:

ma via, dunque, senti e spiegami questo sogno: venti oche qui in casa mi beccano il grano, uscendo dall'acqua, e io mi diverto a vederle. Piombando dal

37. Si badi bene che la scena del dialogo tra Penelope e Odisseo non rimanda per nulla ad un set analitico, pur essendo fondato sul racconto del sogno da parte di lei. Infatti, «l'interprete dei sogni» non è l'equivalente antico dell'analista, giacchè il secondo non imporrebbe mai un'interpretazione propria all'analizzato, visto che è quest'ultimo a dover proporre la sua attraverso le proprie associazioni. Inoltre, essendo sollecitato proprio Ulisse, in questo caso, a fungere da interprete, non si tratterebbe che di un'interpretazione «interessata» e, quindi, di un caso eclatante di scarsa "deontologia professionale». 
monte un'aquila grande, becco adunco, a tutte spezzò il collo e le uccise; riverse giacevano in casa, in un mucchio; poi l'aquila al cielo luminoso s'alzò [...] A un tratto tornando, s'appollaiava sull'orlo del tetto e con parola umana mi tratteneva: mi disse: coraggio figlia del glorioso Icario; non sogno, questa è visione reale, che si avvererà: l'oche i tuoi pretendenti e io t'ero l'aquila prima ma oro torno e sono il tuo sposo legittimo e ai pretendenti tutti darò morte ignobile. (L. XIX, vv. 535-550)

Ma Penelope, durante il sogno, ha pianto e singhiozzato:

E io piangevo e singhiozzavo nel sogno (L. XIX, v. 541)

A questo punto, alcuni aspetti vanno notati. In primo luogo: nessuna divinità ha inviato il sogno dell'aquila e delle oche a Penelope e, dunque, meno che mai l'immagine di qualcuno si è collocata sulla sua testa. In secondo luogo: pur nella sua oscurità, il sogno non costituisce un'esortazione, ma piuttosto una predizione. In terzo luogo: non è possibile distinguere il suo eventuale carattere "predittivo" dallo stesso desiderio di Penelope del ritorno del marito. In quarto luogo: esso è contraddistinto da elementi fortemente simbolici (l'aqui$\mathrm{la}^{38}$ e le oche) che sembrano frutto di metafore fondate su semplici similitudini: in particolare, la forza e la regalità dell'aquila, che altri non è che Odisseo, dopo l'uccisione delle oche, si mette a protezione della dimora. ${ }^{39}$ Infine: l'inconsapevolezza della donna rispetto ad una possibile natura diversa del proprio sogno non appare particolarmente significativa, in quanto anche lei è portatrice di una concezione tradizionale vigente almeno, come si è visto, fino a Platone e Aristotele.

In realtà, appare verosimile sostenere che, in questo caso, la genesi del sogno sia interna e non esterna al soggetto e che, dunque, il soggetto sognatore sia un soggetto "attivo» che proietta il contenuto di una parte del suo animo nel sogno stesso. Cioè come il suo sogno sia prodotto del suo animo, dei desideri confligenti che in esso si agitano, del conflitto interiore tra questi e la realtà più prossima e, infine, dallo stesso conflitto sempre intimo che si genera nell'animo di Penelope tra il desiderio del ritorno di Ulisse e la considerazione che la spinge ad accettare un possibile nuovo matrimonio con uno dei pretendenti. Se non fosse stato elaborato nel settimo secolo a. C., il sogno di Penelope rappresenterebbe, forse, un esempio semplice, ma paradigmatico della concezione moderna dell'attività onirica. ${ }^{40}$

38. L'aquila ha da sempre trovato un posto speciale nelle rappresentazioni simboliche e nell'iconografia. In particolare, nel mondo greco essa costituisce una delle immagini di Zeus, di cui spesso mantiene tra i propri artigli i fulmini. Ricorderò Zeus-Aquila nel rapimento di Ganimede e nel supplizio di Prometeo.

39. A proposito di paragoni, confronti e similitudini in Omero, cfr. Bruno SNELL, op. cit., in particolare il cap. XI.

40. Il Libro XIX risulta essere successivo (VII secolo?) agli altri che compongono il poema, ma è certamente precedente all'epoca di Platone e Aristotele. 
E’ sul desiderio di Penelope-donna che Giulia Sissa costruisce un'argomentazione convincente sullo sviluppo della rappresentazione dell'Io femminile nella Grecia antica. ${ }^{41}$ Se Medea, follemente innamorata e abbandonata da Giasone, ucciderà i figli generati con lui, si collocherà come Achille sul registro dell'eccesso e, specchio femminile dell'eroe pelide, costituirà un punto di continuità nella rappresentazione delle passioni, Penelope è la Moglie legittima e fedele che attende, la Madre premurosa che vuole salvaguardare i diritti di legittimità del figlio Telemaco. Purtuttavia, la donna è dominata da desideri contrastanti:

Così pure il mio cuore con moti opposti s'agita di qua e di là, se restare col figlio e serbare fedelmente ogni cosa, la mia ricchezza, gli schiavi, il palazzo alto e grande, avendo riguardo al letto nuziale e la voce del popolo; oppure ormai seguire tra gli Achei chi è il più nobile, e mi corteggia in palazzo, offrendo doni infiniti. (L. XIX, vv. 524-529)

Il nodo da sciogliere è, dunque, il seguente: restare fedele al marito-padre, oppure scegliere il nuovo marito. Nell'interpretazione data da Sissa, è la prova dell'arco richiesta da Penelope a testimoniare l'affermarsi del desiderio. Del desiderio femminile. Libera dal problema dei beni legittimi assicurati al figlio dalla tradizione patrilineare, e libera da un'eventale pressione paterna, Penelope sceglie una prova fondata sulla fisicità maschile, sui corpi desideranti dei pretendenti: al loro desiderio, risponde con un appello a quegli stessi corpi. ${ }^{42}$ In tal senso, tre brevi considerazioni vanno fatte. La prima: Penelope afferma il proprio Io in quanto la decisione è presa senza un vincolo esterno, meno che mai maschile, in piena autonomia. E', forse, la prima rappresentazione di un Io femminile non etero-diretto, parallelo a quello di Achille che rifiuta i doni di Agamennone, e che non si colloca sul piano dell'ira e dell'eccesso, ma su quello del desiderio femminile. La seconda: il sogno dell'aquila e delle oche sembra essere, proprio per tale ragione e come interpreteremmo oggi, il frutto di due desideri contrastanti che generano conflitto nell'animo di Penelope: l'attesa del marito, verosimilmente morto o comunque distante, la scelta di un nuovo rapporto amoroso.

Se, dunque, il personaggio-Penelope costituisce un'eccezione nel panorama delle donne omeriche e della loro identità, il suo sogno, allora, appare come "eccezionale», molto prossimo ad una concezione moderna dell'onirico e ciò per le ragioni seguenti. In primo luogo: è il puro prodotto della psiche di Penelope, nella quale desideri tra loro confligenti si manifestano. In secondo luogo: esso rappresenta un "compromesso" doloroso tra le spinte divergenti e contraddittorie fra loro. In terzo luogo: come nella teoria freudiana, esso lavora per "spostamento e condensazione», nel senso che allontana i complessi contenuti dei

41. Cfr. Giulia SISSA, Eros tiranno. Sessualità e sensualità nel mondo antico, Bari: Laterza, 2003, in particolare la parte prima, Il desiderio.

42. Giulia SisSA, op. cit. p. 37. 
pensieri soggiacenti (desideri contrastanti, nodi irrisolti ecc.), condensandoli in forma più scarna e semplificata. In quarto luogo: tale lavoro è in tutto analogo a quello previsto nella formazione retorica della metafora. Siano esse fondate su similitudini o non lo siano, le metafore si sviluppano sempre sulla base di tale meccanismo: spostamento di identità su di un elemento di altra natura e condensazione delle qualità e attributi riconosciuti: la dulce boca gongorina ha il bianco splendente delle due file di denti come due fili di perle hanno il bianco splendente, quindi i denti dell'amata sono due fili di perle bianche, la bocca ha per denti due fili di perle e, quindi ancora, la bocca ha due fili di perle. In quinto luogo: esso assume regole logiche di causalità, identità e connessione del tutto diverse da quelle ordinarie. Possono mai le aquile parlare con voce umana, sottraendosi così alla "necessità» cui le costringe la loro natura? Può un uomo avere le ali per volare fin verso il sole ed avvicinarglisi tanto da bruciarsi? Può mai il fegato di un uomo straziato dal becco di un'aquila ogni giorno ogni giorno rigenerarsi? La considerazione della ananke ci spinge a dire no. Ciò è possibile soltanto, infatti, nel Mito o nel sogno. Più che chiedersi se l'uomo chiamato Omero o se gli uomini che hanno condiviso tale nome fossero artefici coscienti di tale mutamento o che ne avessero registrato fedelmente l'affermarsi, sembrerebbe più opportuno interrogarsi sulla semplificazione operata da Freud in relazione al mondo greco pre-filosofico. Forse il padre della psicoanalisi è più legato, come direbbe Giorgio Colli riprendendo Platone, all'epoca dei filosofi che a quella dei sapienti. Forse, non così K. Gustav Jung con la sua attenzione al Mito.

\section{L'Io assente e l'inconscio Es-terno}

In due occasioni, la narrazione omerica presenta due personaggi i cui comportamenti e i cui desideri non possono dirsi sempre e in tutto etero-diretti o etero-governati: Achille e Penelope. Del sogno di Penelope ho qui sopra discusso, di Achille mi è capitato nella precedente occasione qui già citata. A proposito di Achille, però, ricorderò nuovamente come con Vegetti (1995) e Feyerabend (2002) si possa riconoscere nella sua ira, scatenata per essersi visto sottrarre Briseide da Agamennone:

la prima e ancora incerta percezione di sé come soggetto sia pur precariamente unificato di azione avviene dunque nel fuoco dell'emozione collerica, nella reazione violenta ed aggressiva alla minaccia che viene dall'altro. ${ }^{43}$

Il successivo rifiuto dei doni riparatrici di Agamennone costituisce il passaggio finale di una definizione di una soggettività autonoma. Con Citati (2002) si può affermare:

43. Cfr. Paul K. Feyerabend, Conquest of Abundance, University of Cicago Press, trad. it., Conquista dell'abbondanza. Storie dello scontro fra astrazione e riccehezza dell'Essere, Milano: R. Cortina Ed., 2002; e Mario VegETTI, Passioni antiche: l'Io collerico, 1995, in Silvia VegETTI FINZI, op. cit., p. 40. 
Se questa è la prima volta che l'Io prorompe nella letteratura occidentale, si tratta di un'esplosione spaventosa, capace di distruggere il mondo. [...] L'offesa che ha ricevuto è inespiabile: non può venire riparata con doni, scambi di cose, o possessi. Egli non tollera che le passioni del suo io, la sua menis, vengano scambiate con oggetti. ${ }^{44}$ (p. 77 )

In tal senso, le ancora incerte e, soprattutto, irriflesse affermazioni di una soggettività autonoma e di un Io, si legano al mondo delle passioni e della loro rappresentazione linguistica. Nel caso di Achille sul piano della menis e, quindi dell'eccesso, nel caso di Penelope, invece, sul piano del desiderio femminile che, come tale, è fortemente costruito interiormente e manifestato principalmente attraverso il linguaggio verbale e non verbale.

\subsection{L'uomo omerico: membra senza corpo}

Achille e Penelope: le loro rappresentazioni della soggettività e dell'Io appaiono "eccezionali», certamente non a causa dell'inconsapevolezza dei due, ma per l'isolamento della loro affermazione, per l'appunto, della soggettività e dell'Io nei due poemi. Achille la manifesta con l'ira e il rifiuto e di quest'ultimo, interrogato, non potrebbe forse neanche illustrarne le ragioni: esse, forse, sono soltanto un moto profondo che non può essere verbalizzato e reso cosciente. Anche la manifestazione dell'Io di Penelope si presenta per due volte consecutive: la scelta della prova e il sogno. E quest'ultimo trasmette anche l'immagine di un Inconscio (Es) che si mostra attraverso il linguaggio onirico. Ciò giustifica la pertinenza del sogno dell'aquila e delle oche in questo contributo. Inoltre, Achille e, soprattutto, Penelope sono soli: isolati ed eccezionali nel mondo in cui agiscono. Da ciò la loro rilevanza nella tradizione omerica, dal punto di vista della definizione occidentale del Soggetto. Pertanto, essi costituiscono il punto di partenza della nostra narrazione sul di esso.

Achille, e meno che mai Penelope, non sono Agamennone. Si deve ancora a Bruno Snell l'interpretazione di sintesi dei caratteri de «'uomo omerico», sia dal punto di vista del corpo come dell'anima. I corpi umani sono costituiti da mélea kaì guia ("membra con forti muscoli»), cioè non hanno una raffigurazione unitaria. Sono, cioè, un agglomerato di parti distinte, prive di un valore unitario. Lo stesso lessico ne costituisce la manifestazione migliore: soma ("corpo» nel senso di «cadavere»), demas («figura»), kros ("pelle», come superficie), derma («pelle» come involucro staccabile), sono lontani dall'essere "corpo». ${ }^{45}$ Dunque, in questa dimensione ci sono membra legate fra loro, uomini e donne che hanno diversi attributi, uomini «giusti», ad esempio, ma non l'idea di Giustizia. Cioè, si è ancora privi dell'idea stessa di astrazione. Bisognerà, inoltre, attendere l'arte classica del v secolo per registrare un'altra immagine. D'altronde, la fisicità della membra separate appare anche nelle 
rappresentazioni del linguaggio verbale cui si è accennato in precedenza: ad esempio, il suono delle parole che oltrepassa la siepe dei denti. ${ }^{46}$ Analogo discorso vale per «anima». L'anima (psychè) per Omero è soltanto ciò che anima l'uomo. Un soffio vitale, come si è già detto in precedenza, con il riferimento al volume di Giovanni Reale. In ogni caso, a tale riguardo, rimando alla prima parte del saggio di Vegetti (1996). Nel mondo omerico, dunque, non è possibile dire Io sono, poiché è la stessa idea astratta di essere a non sussistere. ${ }^{47} \mathrm{Biso-}$ gnerà attendere, ancora una volta, Patone.

Ciò che qui mi preme sottolineare, invece, è la considerazione seguente: se il mondo omerico, nella sua rappresentazione così come essa ci è giunta, non possiede una concezione unitaria dell'individuo e del suo corpo, e non ha una concezione se non puramente fisica dell'anima, allora non può possedere neanche una concezione della soggettività e, quindi, dell'Io. Naturalmente, è fortemente probabile che, nel percorso che porterà la cultura greca al regime filosofico classico e di qui successivamente al mondo moderno, si siano fatte spazio lentamente e in modo irriflesso e inconsapevole visioni, più che alternative, complementari. Visioni certamente marginali all'inizio, condivise successivamente e ancor'oggi presenti.

\subsection{Il ruolo della rappresentazione della divinità: spostamento e condensazione}

Ah quante colpe fanno i mortali agli dei! Da noi dicon essi che vengono i mali, ma invece dei loro folli delitti contro il dovuto han dolori (Odissea, L. I, vv. 32-34)

E' Zeus ${ }^{48}$ stesso a suggerire le conclusioni cui è possibile qui pervenire. Gli dei sono i governanti dell'animo umano? Da loro discendono negli uomini e nelle donne passioni, desideri, emozioni, regole etiche? E i sogni? Per quanto possano ritenerlo le tradizioni e le narrazioni che di Omero portano il nome, è lo stesso Sovrano dell'Olimpo a smentirle. Anche se, come è ovvio, le rappresentazioni discorsive e le concezioni soggiacenti citate tendono inerzialmente a riprodursi e a restare vigenti a lungo. In rappresentazioni di tale tipo, pertanto, sembra operare un meccansimo di spostamento e di condensazione, in tutto analogo a quello commentato qui in relazione alla metafora e al sogno.

\section{Cfr. Anna Marina Storoni Piazza, op. cit.}

47. Per pure necessità di spazio non posso qui riprendere l'argomentazione di Havelok sulla nascita del verbo einai «essere»), che ritengo potersi presentare a sostegno dell'analisi qui costruita. Rimando pertanto a Henry A. HAVELOCK, The Greek Concept of Justice from Its Shadows in Homer to Its Substance in Plato, Cambridge: Harvard Univ. Press, (Mass.), 1978, trad. it.: Dike. La nascita della coscienza, Bari: Laterza, 1981; in particolare il cap. XIII.

48. Ricorderò qui che Zeus, contrariamente ad altre divinità indoeuropee analoghe, non solo non è creatore dell'universo, ma non rientra neanche nel gruppo delle divinità greche primordiali. Nonostante debba lasciare il dominio dell'oceano a Poseidone e quello del mondo sotterraneo ad Ade, Zeus signore del cielo si mostra il capo incontestato degli dei e signore assoluto dell'universo. Per tali questioni cfr. Mircea ELIADE, Histoire des croyances et des idées réligieuses, Paris: Payot, 1975; trad. it.: Storia delle credenze e delle idee religiose, Firenze: Sansoni, 1990. 
Tale operazione sembra poter dare coerenza al tutto e meglio illustrare, in particolare, la concezione stessa del sogno che Freud definiva "primordiale».

Se, infatti, in greco omerico, non è possibile dire Io sono e, dunque, non ci può essere coscienza e Io, allora non è possibile elaborare neppure una visione anche primitiva dell'Inconscio, dell'Es. Ma la natura "demoniaca», o meglio quella parte complessa e perennemente in ombra dell'animo umano, che genera conflitti tra desideri che si scontrano, tra desiderio e realtà, pur continua ad esistere. Altra strada non c'è che quella della proiezione - dello spostamento- all'esterno, da cui essa ritornerà mascherata e deformata - condensata e semplificata - nell'immagine stessa delle divinità. Divinità incolpevoli, ma pur tuttavia identificate con essa.

Un Es (es)terno dovuto all'assenza della coscienza di un Io. Ciò è quanto consente all'umanità omerica di ritenere che la divinità sia fonte delle proprie emozioni, passioni e desideri — anche inconfessabili- così come dei propri sogni: anche il sogno è da Zeus [Iliade, Libro I, v, 63]. Dunque: sogni che l'umanita del tempo non può fare altro che vedere. 